\title{
Challenges of Managing Emergency Ileostomy: Nutrition-A Neglected Aspect
}

\author{
Ravindra Singh Mohil, ${ }^{1}$ Nitisha Narayan, ${ }^{1}$ S. Sreenivas, ${ }^{1}$ Namrata Singh, ${ }^{2}$ \\ Abhinav Bansal, ${ }^{1}$ and Gulshan Jit Singh ${ }^{1}$ \\ ${ }^{1}$ Department of Surgery, Safdarjang Hospital, Dr. V. M. Medical College, New Delhi 110023, India \\ ${ }^{2}$ Department of Gastroenterology and Human Nutrition, AIIMS, New Delhi 110029, India \\ Correspondence should be addressed to Ravindra Singh Mohil, rsmohil@gmail.com
}

Received 1 October 2012; Accepted 1 November 2012

Academic Editors: C. C. Chang, E. De Guise, and S. Di Saverio

Copyright ( 2012 Ravindra Singh Mohil et al. This is an open access article distributed under the Creative Commons Attribution License, which permits unrestricted use, distribution, and reproduction in any medium, provided the original work is properly cited.

\begin{abstract}
Objective. The present study was carried out with an aim to study the nutritional status of patients undergoing emergency ileostomy using simple bedside tools in a developing country. Methods. Nutritional assessment (anthropometry, biochemical, immunological, and dietary) was done within 24-36 hours of admission and 6 weeks postoperatively. Primary endpoint was the study of the nutritional status of the patients with ileostomy. Results. $N=58,47$ males with mean age 32 years. Postoperatively 50 $(86.2 \%)$ patients experienced some complications including those related to ileostomy. Malnutrition varied from 7 (12.1\%) using BMI to $54(93.1 \%)$ by triceps skinfold thickness. At 6 weeks, despite adequate nutritional intake, there was a significant decrease in almost all nutritional parameters except serum albumin which was normal in most patients. Factors contributing to weight loss in $41(70.7 \%)$ patients were decreased length of proximal bowel left $(P=0.001)$, increased ileostomy output $(P=0.001)$, delayed surgery $(P=0.004)$, and increased disease severity score $(P=0.005)$. Conclusion. Majority of patients undergoing emergency ileostomy were malnourished and had significant nutritional depletion despite adequate nutritional support. Serial assessment helps to assess nutritional recovery in these patients.
\end{abstract}

\section{Introduction}

Ileal perforation peritonitis continues to be a common surgical emergency in developing world. In India, unlike the western countries where tuberculosis and typhoid are almost unknown, these continue to be endemic and are the most commonly seen causes of ileal perforation [1]. Construction of an ileostomy is often done in patients with ileal perforation who are very toxic, having gross fecal contamination, multiple/large perforation or unhealthy bowel [2].

Construction of an ileostomy inevitably results in an increased fecal loss of fluids and electrolytes with a reduction in total body water and has been interpreted as a state of chronic dehydration [1]. In addition to this, these patients often have an inappropriate food intake and are frequently in negative energy balance leading to malnutrition, impaired immunity, and deranged bodily functions [3]. Even a modest resection of the terminal ileum undertaken during the course of proctocolectomy decreased body weight largely because of a reduction in body fat [4]. It is important for healthcare professionals involved in the care of patients with an ileostomy to appreciate that stoma surgery changes the body's usual processes of nutritional absorption and excretion, so that informed support and advice on diet can be provided [5].

The construction of ileostomies in ileal perforations in the setting of ileal perforations prevalent in resource crunched developing countries bears a special attention as the morbidity is high owing to the existing disease process, sepsis, and malnourishment $[2,4]$.

General surgeons usually find nutritional assessment very difficult especially in those undergoing emergency laparotomy and often omit this during the management of the patients. The present study was therefore carried out with an aim to study the nutritional status of the patients with ileostomy using simple bed side tools (anthropometry, biochemical, immunological, and dietary) in these patients. 


\section{Materials and Methods}

The study was conducted in the surgical ward of a tertiary care, free, government hospital in the capital city of a developing country over a period of 18 months. The study was conducted according to the guidelines laid down in the Declaration of Helsinki and was approved by the hospital ethics committee. A written and witnessed verbal informed consent was obtained from all patients and formally recorded in the case sheet. All patients who presented in the emergency in a single surgical unit with ileal perforation peritonitis were included in the study. All patients were enrolled postoperatively in the study. All adult patients more than 12 years of age undergoing emergency ileostomy were included in the study. On admission a detailed clinical examination related to the main disease along with nutritional assessment was carried out.

\subsection{Assessment of Disease Severity and Operative Findings.} Disease severity (physiological and operative score) was calculated using Physiologic and Operative Severity Scoring for the enumeration of Mortality and Morbidity-POSSUM [6], its variant Portsmouth P-POSSUM [7], and APACHE II [8] scores at the time of admission. Predicted morbidity and mortality scores were calculated based on physiologic and operative scores using POSSUM and P-POSSUM equations as under:

2.1.1. POSSUM. Morbidity- $\log [R /(1-R)]-5.91+(0.16$ $\times$ physiological score $)+(0.19 \times$ operative severity score $)$.

Mortality- $\log [R /(1-R)]-7.04+(0.13 \times$ Physiological Score $)+(0.16 \times$ operative severity score $)$.

2.1.2. P-POSSUM. Mortality-Log $[R /(1-R)]-9.065+$ $(0.1692 \times$ Physiological Score $)+(0.1550 \times$ operative severity score).

Analysis was performed using exponential method for POSSUM and linear for P-POSSUM for correctly predicting the number of death and complications $[9,10]$. Any mortality and morbidity within a period of 30 days were also noted as per the definitions of POSSUM score defined by Copeland et al. [6].

APACHE-II. Severity scoring was also done as per the APACHE score, and any morbidity and mortality were observed based on the severity of the APACHE II score [8].

The nutritional assessment was performed by a single investigator part of the surgical team after adequate training within 24-36 hours of admission using different nutritional indices. This included a detailed history, anthropometry, biochemical, and immunological assessment. The assessment was repeated at 6 weeks postoperatively. The nutritional history was done as per the set pattern for all patients with the help of proforma on the basis of semiquantitative food frequency questionnaire.

Anthropometry using standard techniques included height, weight, body mass index (BMI), mid-arm circumference (MAC), and triceps skinfold thickness (TSF).
Mid-arm muscle circumference (MAMC) was derived from the equation MAMC $=$ MAC $-\pi \times$ TSF. Biochemical included haemoglobin, serum albumin, and creatinine height index (CHI). From the estimated 24 hrs urinary creatinine excretion, $\mathrm{CHI}$ was calculated using the original standards of Bristrian [11]. Immunological parameters included total lymphocyte count (TLC) derived by multiplying total leucocyte count with percent lymphocyte count and delayed cutaneous hypersensitivity (DH). Purified protein derivative (PPD) $5 \mathrm{TU} / 0.1 \mathrm{~mL}$ was injected intradermally on the ventral aspect of forearm to measure delayed hypersensitivity response at 48 hours. The anthropometric values were compared with percentages of normal values for age and sex. TSF values $<12.5 \mathrm{~mm}$ for males and $<16.5 \mathrm{~mm}$ for females were taken as malnourished. The cut-off value for MUAC was taken as $26.4 \mathrm{~cm}$ for males and $25.7 \mathrm{~cm}$ for females. The standard values for MAMC were $25.3 \mathrm{~cm}$ for males and $23.2 \mathrm{~cm}$ for females. Values were categorized as severe if $<60 \%$ of standard, moderate $61-80 \%$ of standard, and mild when $81-90 \%$ of standard. Values $>90 \%$ of standard were considered normal $[12,13]$. Body Mass Index (BMI) which is derived by dividing body weight with height in meter square is a good indicator for nutritional assessment. It is increasingly being used for assessing and grading of nutritional status. Values $<18.5$ were categorized as malnourished; 18.5 to 25 were taken as normal, and those with $>25$ were labeled as overweight [14]. Haemoglobin values in adult males of $<12 \mathrm{gm} \%$ and $<11.5 \mathrm{gm} \%$ in females were considered anaemic based on standard values [15]. Total lymphocyte count $>2000 / \mathrm{mm}^{3}$ was considered normal, between $1200-1999 / \mathrm{mm}^{3}$ as mild depletion, 800 1199 as moderate depletion, and those $<800$ as severely depleted [16]. Serum albumin $>3.5 \mathrm{gm} \%$ was considered normal. Concentrations between 2.8-3.5 gm\%, between 2.1$2.7 \mathrm{gm} \%$ and $<2.1 \mathrm{gm} \%$, were categorized as mild, moderate, and severe depletion, respectively [16]. Standards laid down by Bristrian were followed to calculate creatinine height index (CHI) [11]. The response to intradermal injection of PPD antigen was evaluated at $48 \mathrm{hrs}$ after the test dose. Any induration $>5 \mathrm{~mm}$ and an erythema of more than 15-20 mm were considered positive [17]. Response other than this was considered negative. Other tests to assess organ dysfunction included kidney function tests (blood urea and serum creatinine), liver function tests (serum bilirubin, liver enzymes AST, ALT, and alkaline phosphatase), and coagulation profile (bleeding time clotting time, prothrombin time, and PTTK).

Postoperatively patients were taught to record his daily diet and ileostomy output in a diary. The patients were counseled to take small frequent meals and adequate fluid intake, and a individualized diet chart was formulated for each patient using Harris-Benedict equation. Each patients intake was strictly monitored, and all patients were regularly reviewed for the compliance with diet. Seventeen patients were readmitted for dehydration, burst abdomen, and anastomotic leaks. Followup was done at six weeks with repeat measurement of the same physical and biochemical parameters. 
TABLE 1: Indications for emergency ileostomy $(n=58)$.

\begin{tabular}{lc}
\hline Diagnosis & $N(\%)$ \\
\hline Enteric perforation & $25(43.1 \%)$ \\
Tubercular ileal perforation & $17(29.3 \%)$ \\
Gangrenous bowel & $11(18.9 \%)$ \\
Anastomotic leak & $2(3.4 \%)$ \\
Traumatic perforation & $1(1.7 \%)$ \\
\hline
\end{tabular}

TABLE 2: Postoperative complications encountered.

\begin{tabular}{lc}
\hline Complications & No. of patients (\%) \\
\hline Wound infection & $44(75.8 \%)$ \\
Wound dehiscence & $33(56.9 \%)$ \\
Chest infection & $23(39.6 \%)$ \\
Urinary tract infection & $11(18.9 \%)$ \\
Respiratory failure & $8(13.8 \%)$ \\
Renal failure & $5(8.62 \%)$ \\
Cardiac failure & $3(5.2 \%)$ \\
Anastomotic leak & $2(3.4 \%)$ \\
Skin excoriation & $36(62.1 \%)$ \\
Dehydration & $17(29.3 \%)$ \\
Prolapse & $9(15.5 \%)$ \\
Proximal perforation & $3(5.2 \%)$ \\
Retraction of stoma & $1(1.7 \%)$ \\
\hline
\end{tabular}

\section{Results}

A total of 75 consecutive patients undergoing emergency ileostomy were assessed with 58 of them actually being included in the study. Of the seventeen patients who were excluded, twelve died before six weeks, one left against medical advice, two were lost to followup, and two underwent reexploration following surgery done outside. $\mathrm{M}: \mathrm{F}-47: 11$, with mean age of 32.13 (15-68) years. The indications for surgery in this group of patients are shown in Table 1. Fortyfive patients had a loop and 13 end ileostomy with enteric perforation and Koch's abdomen being the commonest cause for emergency laparotomy. Postoperatively, 50 (86\%) patients experienced one or more complication, including those specific to ileostomy and are shown in Table 2.

Three patients developed proximal perforation despite ileostomy being done to prevent anostamotic leak. All these underwent reexploration, and none of the patient died.

The average proximal bowel left was $497.22 \mathrm{~cm}(150 \mathrm{~cm}-$ $585 \mathrm{~cm}$.) with mean output of $3.5 \mathrm{~L} / 24 \mathrm{hrs}(1.5 \mathrm{~L}-4.5 \mathrm{~L} /$ $24 \mathrm{hrs}$ ) in the first week of construction of the ileostomy. As expected, output was higher in initial postoperative period and patients with a shorter length of proximal bowel. The output gradually decreased at 6 weeks, and the content became thicker as the patients were started on a more solid diet and bulk forming foods. Seventeen patients who got readmitted as a result of severe dehydration were found to have an inadequate fluid and salt intake although their $24 \mathrm{hr}$ calorie intake was normal. They were treated with I/V fluid replacement and advised to take ORS solution.
3.1. Nutritional Assessment: Anthropometry. On initial nutritional assessment, it was found that the percentage of malnourished patients varied when different parameters were used for nutritional assessment. Seven $(12.06 \%)$ patients had a BMI $<18.5$ and were malnourished, $49(84.48 \%)$ patients had normal BMI, and only two patients (3.44\%) were overweight at admission. Based on various other parameters, the degree of malnutrition varied, ranging between $6 \%$ (MAMC) to 93\% (TSF) (Table 3).

3.2. Biochemical Parameter. Serum albumin and total lymphocyte count were lower than normal in $84 \%$ and $86 \%$ of the patients, respectively, on initial assessment. A comparison of nutritional assessment using various nutritional parameters is shown in Table 3.

Due to absence of Indian standards and using western standards for assessment; majority of our patients had a very low muscle and fat mass. Based on creatinine height index (CHI) $43.10 \%$ patients had mild and moderate protein deficiency each and $13.79 \%$ had severe protein deficiency at presentation. At 6 weeks more than half $(51.76 \%)$ had severe to moderate protein deficiency. (how many now modsevere). Thirty-one (53\%) patients were anaemic, $\mathrm{M}=24$ (51\%) and $\mathrm{F}=7(63 \%)$ while at 6 weeks $41(70.8 \%)$ were anemic; $\mathrm{M}=32(78 \%)$ and $\mathrm{F}=9(22 \%)$. Mantoux reaction was negative at 48 hours in $24(42 \%)$ of the patients.

Disease severity of the patients was studied by using POSSUM, P-POSSUM and APACHE-II scores. Mean POSSUM morbidity, mortality, P-POSSUM \& APACHE score were $73.83,50.75,29.43$ and 9 respectively and the number of complications increased as the mean score increased. Patients with APACHE-II scores between 0-10 showed $82.6 \%$ morbidity, those with scores above 10 had $100 \%$ morbidity.

3.3. Change in Nutritional Status. After discharge the average $24 \mathrm{hr}$ calorie, protein, carbohydrates, and fat intake was $1852.33 \mathrm{Kcal}, 59.33 \mathrm{gms}, 61.2 \mathrm{gms}$, and 266 gms, respectively. (by recall method and diary kept by the patient). There was a statistically significant decrease in almost all the nutritional parameters at the end of six weeks except for TLC, CHI, and serum albumin (Table 4). Though the last three parameters showed a downward trend this did not reach statistical significance. At the end of 6 weeks, $41(70 \%)$ patients had severe weight loss and lost more than $7.5 \%$ of their admission weight.

In fact $43 \%$ (25) patients had lost $>10 \%$ of their admission weight at the end of six weeks. Factors which significantly lead to this weight loss were the amount of proximal bowel left, ileostomy output, age of the patient, delay in reporting to emergency, hemoglobin levels, and POSSUM (disease) severity score (Table 5).

\section{Discussion}

Temporary Ileostomy in emergency setting is usually done in patients presenting with ileal perforation peritonitis. Management of these cases postoperatively poses a challenge 
TABLE 3: Initial nutritional assessment by various nutritional parameters $(n=58)$.

\begin{tabular}{|c|c|c|c|c|}
\hline & SEVERE $<60 \%$ & MODERATE $61-80 \%$ & MILD 81-90\% & NORMAL $>90 \%$ \\
\hline $\operatorname{MAMC}(\mathrm{cm})$ & $5(8.6 \%)$ & $5(8.6 \%)$ & $8(14 \%)$ & $40(69 \%)$ \\
\hline $\operatorname{MUAC}(\mathrm{cm})$ & 0 & $3(5 \%)$ & $1(2 \%)$ & $54(93 \%)$ \\
\hline$(\mathrm{TSF})(\mathrm{mm})$ & $8(14 \%)$ & $30(51.7 \%)$ & $16(27.58 \%)$ & $4(6.8 \%)$ \\
\hline S. Albumin $(\mathrm{gm} / \mathrm{dL})$ & $2(3.44 \%)$ & $3(52 \%)$ & $44(75.8 \%)$ & $9(15.5 \%)$ \\
\hline Total lymphocyte count & $8(13.79 \%)$ & $18(31.03 \%)$ & $24(41.37 \%)$ & $8(13.8 \%)$ \\
\hline $\mathrm{CHI}$ & $8(13.79 \%)$ & $25(43.10 \%)$ & $25(43.10 \%)$ & 0 \\
\hline
\end{tabular}

TABLE 4: Change in the nutritional profile.

\begin{tabular}{lccc}
\hline & $\begin{array}{c}\text { Initial assessment } \\
(\text { Mean } \pm \mathrm{SD})\end{array}$ & $\begin{array}{c}\text { At 6 weeks } \\
(\text { Mean } \pm \mathrm{SD})\end{array}$ & $P$ value \\
\hline BMI $\left(\mathrm{kg} / \mathrm{m}^{2}\right)$ & $20.7 \pm 2.5$ & $18.5 \pm 2.6$ & 0.001 \\
TSF $(\mathrm{mm})$ & $9 \pm 1.7$ & $8.3 \pm 1.1$ & 0.001 \\
MUAC $(\mathrm{cm})$ & $29.1 \pm 3.1$ & $26.5 \pm 2.7$ & 0.001 \\
MAMC $(\mathrm{cm})$ & $23.9 \pm 6.3$ & $23.9 \pm 2.5$ & 0.921 \\
S. Albumin $(\mathrm{g} / \mathrm{dL})$ & $3.1 \pm 0.3$ & $3.1 \pm 0.3$ & 0.796 \\
Hemoglobin $(\mathrm{g} / \mathrm{dL})$ & $11.7 \pm 1.4$ & $10.8 \pm 1.9$ & 0.001 \\
Total lymphocyte count & $1477 \pm 883$ & $1243 \pm 427$ & 0.038 \\
CHI & $55.2 \pm 15.7$ & $53.9 \pm 15.1$ & 0.220 \\
\hline
\end{tabular}

TABle 5: Factors related weight loss $(n=41)$ at six weeks after ileostomy.

\begin{tabular}{lc}
\hline Parameter & $P$ value \\
\hline Age & 0.078 \\
Delay in surgery (in hrs) & $0.004^{*}$ \\
Bowel left & $0.001^{*}$ \\
Ileostomy output & $0.001^{*}$ \\
Hemoglobin & $0.051^{*}$ \\
MUAC & 0.487 \\
TSF & 0.928 \\
S. Albumin & 0.363 \\
Total lymphocyte count & 0.885 \\
CHI & 0.687 \\
Calorie intake & 0.315 \\
Protein intake & 0.304 \\
Fat intake & 0.114 \\
Carbohydrate intake & 0.739 \\
POSSUM & $0.005^{*}$ \\
APACHE-II & 0.287 \\
\hline
\end{tabular}

*Significant.

as they suffer from multiple problems related to ileostomy and the primary disease itself leading to high morbidity [18]. Creation of stoma is associated with high costs for patients and society alike. As reported in various studies, our patients also experience a reduced quality of life due to stoma-associated complications in the form of skin irritation, diarrhoea, prolapse, retraction, parastomal hernia, ileus, and so forth $[19,20]$. Besides feelings of physical and mental restrictions many cases experienced postoperative chest infections (39.6\%) and dehydration (29.3\%); due excess fluid loss requiring readmission was also common and similar to observations by other researchers [21-24].

Nutritional depletion occurs in patients with ileostomy due to both anatomical and functional loss of the gut, ongoing inflammatory activity due to disease with sepsis, preexisting malnutrition, and the added surgical stress. These factors may be contributing in varying proportions in these patients mandating an appropriate management plan to optimize recovery [25].

Unfortunately not much has been reported on the nutritional impact of ileostomy done in emergency, especially in those who are already malnourished with no nutritional standards to compare. In the present study we employed various parameters to study the initial nutritional assessment. The choice of parameters was decided by the fact that all these measurements were simple to perform and required no elaborate equipment, and the results provided a fairly accurate estimate of all the components of the body after a single estimation.

At presentation although patients were sick for any detailed nutritional assessment at the time of admission it could be done within 2-3 days of admission in all the patients included in the study. The prevalence of malnutrition based on anthropometric values varied based on the parameter used, ranging from $12 \%$ using BMI to $93 \%$ based on TSF. Almost 30\% had low muscle mass measure by MAMC which increased to $100 \%$ based on CHI. The value of initial CHI showing muscle depletion could not be considered reliable in the present study as it was done after the surgery which leads to increased muscle breakdown. As the inflammatory activity decreased but not completely at the end of 6 weeks, CHI showed a downward trend though it is was not significant. $\mathrm{CHI}$ assesses only protein deficiency and is therefore of limited value but was used as it was relatively easy to measure and did not add extra costs. Amount of adipose tissue can determine the duration of successful starvation during which body cell mass (BCM) and proteins can be spared and consequently influencing survival. This was depleted in almost $93 \%$ of our patients even at presentation.

These findings may suggest a greater prevalence of undernutrition in the community, but most of our patients were apparently healthy before the disease process started. Ideally malnutrition should be defined based on cutoff values at which greater complications occur [26]. Unfortunately techniques to measure various body compartments (FFM, FM, TSF, and MAMC) have not been very well validated, and 
there is very limited data in surgical patients of these parameters from developing countries. Also the body composition varies between various races, ethnicities, and regions. In the present study, patients were sick for variable periods of time before seeking medical help, ileostomy was done more often in sicker patients, and nutritional assessment could be done up to 2-3 days of surgery in many patients [2]. Combined these factors changed various anthropometric values especially body weight and composition in a short period of time. All these factors may have contributed to this high level of undernutrition and limited the role of anthropometry to make any conclusions in the present study.

Various studies have shown that reduction in the active absorptive area of the small bowel has led to changes in nutritional status with respect to body composition [4]. Even a modest resection of the terminal ileum decreases body weight because of a reduction in body fat as there is increased faecal loss of bile acid leading to malabsorption of fat [27]. In spite of ensuring early adequate nutritional and fluid intake, there was significant nutritional depletion in our patients reflected in various nutritional parameters. At the end of 6 weeks, $41(70 \%)$ patients had severe weight loss as per the ASPEN criteria [28] and lost more than $7.5 \%$ of their admission weight. This cannot be entirely attributed to nutritional depletion as the patients were taking adequate calories and could have been due to negative fluid balance leading to shrinking extracellular water, losses from the ileostomy, or inadequate fluid intake, seen in 17 patients who were readmitted due to dehydration. Besides this as the patient recovers from stress and sepsis excess water in the extracellular compartment is excreted contributing to this weight loss. It was observed that delay in surgery, disease severity/inflammatory activity (POSSUM score), amount of proximal bowel left, and the ileostomy output significantly contributed to this weight loss. Besides weight loss, there was also depletion in fat as well as muscle mass reflected in decreasing BMI, TSF, MAMC, and MUAC values. Thus patients undergoing emergency ileostomy have many adverse features contributing to nutritional depletion which cannot be reversed by nutritional support alone.

Serum albumin and to certain extent hemoglobin levels which have been traditionally considered as nutritional markers are now considered more as an inflammatory marker. Both parameters were on the lower side at presentation in majority of the studied patients [26]. Delayed presentation (mean 2.5 days) could have contributed to this since rapid decrease in albumin levels can occur within 24 hours due to severe catabolism induced by sepsis [26]. At the end of six weeks once the patients recovered from the initial insult, serum albumin normalized in a number of patients. Hemoglobin estimations revealed a significant decrease of $14 \%$ from the initial value at admission although all the patients in the study group were started on oral iron supplements and multivitamins at the earliest after surgery. The loss of functional terminal ileum could have affected anemia in multiple ways along with ongoing inflammation and loss of body cell mass (BCM). This reinforces the fact that both albumin and to certain extent hemoglobin are inflammatory markers. Immunological assessment in our study using total lymphocyte counts revealed $87 \%$ patients to be depleted. Lymphocytopenia in the malnourished state has been reported in a large series of patients [29]. The significance of this is limited because this depletion is not a graded response [26].

Despite the limitation of small sample size and lack of anthropometric and biochemical standards contributing to these results, initial assessment was still useful. It helped us by obtaining baseline values to which subsequent change at the end of six weeks could be compared. However, there is a need to generate larger data base especially in surgical patients to define cutoffs which are more appropriate to a given population especially in developing country like ours.

Closure of ileostomy is being advocated as early as two weeks; to avoid complications of ileostomy may not be ideal in these patients [30,31]. Early second surgery would increase the surgical stress on these patient, and a more delayed reversal could only prolong the morbidity. Even at the end of six weeks many of our patients had not recovered from the impact of surgery despite adequate nutritional support as catabolism, and not the inadequate intake, seemed to be the real challenge and to our ability to provide appropriate nourishment [32]. We offered successful ileostomy closure in all our patients once they showed signs of recovery as assessed by improved appetite, improving performance status, total resolution of sepsis, weight gain, healing of wounds, increasing serum albumin, and hemoglobin along with other nutritional parameters.

To conclude, in developing country like ours ileostomy is being done in emergency as a life-saving procedure in large number of patients mainly from the underprivileged section of the society. Nutrition plays a key role in the management of these patients: serial nutritional assessment (despite its limitation) in assessing recovery and nutritional support to optimize patient before ileostomy closure.

\section{References}

[1] B. Ojerskog, H. Andersson, I. Bosaeus, H. Brevinge, and L. O. Nilsson, "Total body water and total body potassium in ileostomy patients before and after conversion to the continent ileostomy," Gut, vol. 29, no. 9, pp. 1198-1201, 1988.

[2] R. S. Mohil, T. Singh, S. Arya, and D. Bhatnagar, "Risk adjustment is crucial in comparing outcomes of various surgical modalities in patients with ileal perforation," Patient Safety in Surgery, vol. 2, article 31, 2008.

[3] I. Warnold, T. Falkheden, B. Hulten, and B. Isaksson, "Energy intake and expenditure in selected groups of hospital patients," American Journal of Clinical Nutrition, vol. 31, no. 5, pp. 742749, 1978.

[4] J. C. Cooper, A. Laughland, E. J. Gunning, L. Burkinshaw, and N. S. Williams, "Body composition in ileostomy patients with and without ileal resection," Gut, vol. 27, no. 6, pp. 680-685, 1986.

[5] J. Fulham, "Providing dietary advice for the individual with a stoma," British Journal of Nursing, vol. 17, no. 2, pp. S22-S27, 2008.

[6] G. P. Copeland, D. Jones, and M. Walters, "POSSUM: a scoring system for surgical audit," British Journal of Surgery, vol. 78, no. 3, pp. 355-360, 1991. 
[7] M. S. Whiteley, D. R. Prytherch, B. Higgins, P. C. Weaver, and W. G. Prout, "An evaluation of the POSSUM surgical scoring system," British Journal of Surgery, vol. 83, no. 6, pp. 812-815, 1996.

[8] W. A. Knaus, E. A. Draper, D. P. Wagner, and J. E. Zimmerman, "APACHE II: a severity of disease classification system," Critical Care Medicine, vol. 13, no. 10, pp. 818-829, 1985.

[9] L. D. Wijesinghe, T. Mahmood, D. J. A. Scott, D. C. Berridge, P. J. Kent, and R. C. Kester, "Comparison of POSSUM and the Portsmouth predictor equation for predicting death following vascular surgery," British Journal of Surgery, vol. 85, no. 2, pp. 209-212, 1998.

[10] R. S. Mohil, D. Bhatnagar, L. Bahadur, Rajneesh, D. K. Dev, and M. Magan, "POSSUM and P-POSSUM for risk-adjusted audit of patients undergoing emergency laparotomy," British Journal of Surgery, vol. 91, no. 4, pp. 500-503, 2004.

[11] B. R. Bistrian, G. L. Blackburn, J. Vitale, D. Cochran, and J. Naylor, "Prevalence of malnutrition in general medical patients," Journal of the American Medical Association, vol. 235, no. 15, pp. 1567-1570, 1976.

[12] D. D. Henrud, "Nutritional screening and assessment," Medical Clinics of North America, vol. 83, pp. 1525-1546, 1999.

[13] I. Giovanni and G. Boldrini, "RQ and patterns of substrate utilization in human sepsis and trauma," Journal of Parenteral and Enteral Nutrition, vol. 7, p. 226, 1983.

[14] D. G. Kelly, S. F. Phillips, and K. A. Kelly, "Dysfunction of the continent ileostomy: clinical features and bacteriology," Gut, vol. 24, no. 3, pp. 193-201, 1983.

[15] K. V. Krishna Das, "Nutritional anaemias in India," The Journal of the Association of Physicians of India, vol. 28, no. 12, pp. 521-533, 1980.

[16] J. P. Grant, P. B. Custer, and J. Thurlow, "Current techniques of nutritional assessment," Surgical Clinics of North America, vol. 61, no. 3, pp. 437-463, 1981.

[17] D. L. Palmer and W. P. Reed, "Delayed hypersensitivity skin testing-II. Clinical correlates and anergy," Journal of Infectious Diseases, vol. 130, no. 2, pp. 138-143, 1974.

[18] D. P. O'Leary, C. J. Fide, C. Foy, and M. E. Lucarotti, "Quality of life after low anterior resection with total mesorectal excision and temporary loop ileostomy for rectal carcinoma," British Journal of Surgery, vol. 88, no. 9, pp. 1216-1220, 2001.

[19] I. Robertson, E. Leung, D. Hughes et al., "Prospective analysis of stoma-related complications," Colorectal Disease, vol. 7, no. 3, pp. 279-285, 2005.

[20] E. Carlsen and A. B. Bergan, "Loop ileostomy: technical aspects and complications," European Journal of Surgery, vol. 165, no. 2, pp. 140-144, 1999.

[21] M. D. Sher-uz-Rehman, S. Atiq-ur-Rehman, H. Famad, and K. Younis, "Loop ileostomy: complications in cases of enteric perforations," Professional Medical Journal, vol. 18, no. 2, pp. 222-227, 2011.

[22] H. R. Roy, M. A. Basunia, and M. A. Quazum, "Complications of protective ileostomy in Emergency surgery-a study of 50 cases," Journal of Bangladesh College of Physicians and Surgeons, vol. 29, no. 4, 2011.

[23] M. Sutters, D. J. S. Carmichael, R. J. Unwin et al., "“Low sodium" diuresis and ileal loss in patients with ileostomies: effect of desmopressin," Gut, vol. 32, no. 6, pp. 649-653, 1991.

[24] M. M. G. Rathnayake, S. K. Kumarage, S. R. E. Wijesuriya, B. N. L. Munasinghe, M. H. J. Ariyaratne, and K. I. Deen, "Complications of loop ileostomy and ileostomy closure and their implications for extended enterostomal therapy: a prospective clinical study," International Journal of Nursing Studies, vol. 45, no. 8, pp. 1118-1121, 2008.
[25] R. S. Mohil, A. Agarwal, N. Singh, J. Arora, and D. Bhatnagar, "Does nutritional status play a role in patients undergoing emergency laparotomy?" e-SPEN, vol. 3, no. 5, pp. e226-e231, 2008.

[26] P. B. Soeters, P. L. M. Reijven, M. A. E. van Bokhorst-de van der Schueren et al., "A rational approach to nutritional assessment," Clinical Nutrition, vol. 27, no. 5, pp. 706-716, 2008.

[27] L. O. Nilsson, H. Andersson, L. Hulten et al., "Absorption studies in patients 6 to 10 years after construction of ileostomy reservoirs," Gut, vol. 20, no. 6, pp. 499-503, 1979.

[28] G. L. Blackburn, B. R. Bistrian, B. S. Maini, H. T. Schlamm, and M. F. Smith, "Nutritional and metabolic assessment of the hospitalized patient," Journal of Parenteral and Enteral Nutrition, vol. 1, no. 1, pp. 11-22, 1977.

[29] A. N. Naidu and N. P. Rao, "Body mass index," European Journal of Clinical Nutrition, vol. 48, no. 3, pp. 153-159, 1994.

[30] T. Hindenburg and J. Rosenberg, "Closing a temporary ileostomy within two weeks," Danish Medical Bulletin, vol. 57, no. 6, article A4157, 2010.

[31] W. Baraza, J. Wild, W. Barber, and S. Brown, "Postoperative management after loop ileostomy closer: are we keeping patient in hospital too long?" Annals of the Royal College of Surgeons of England, vol. 92, no. 1, pp. 51-55, 2010.

[32] D. W. Wilmore, "Metabolic response to severe surgical illness: overview," World Journal of Surgery, vol. 24, no. 6, pp. 705-711, 2000. 


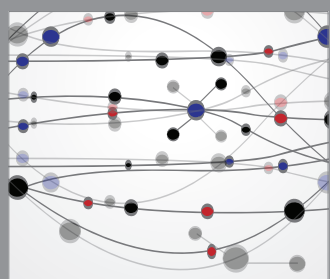

The Scientific World Journal
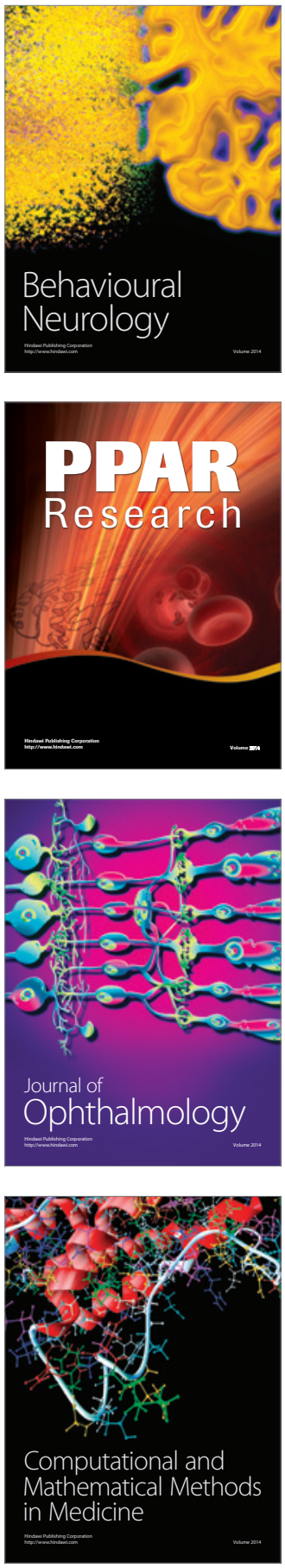

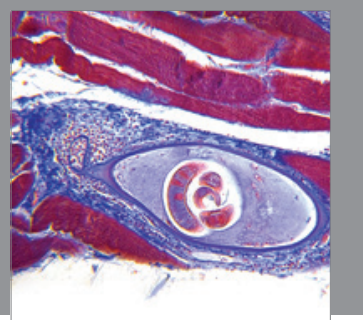

Gastroenterology

Research and Practice
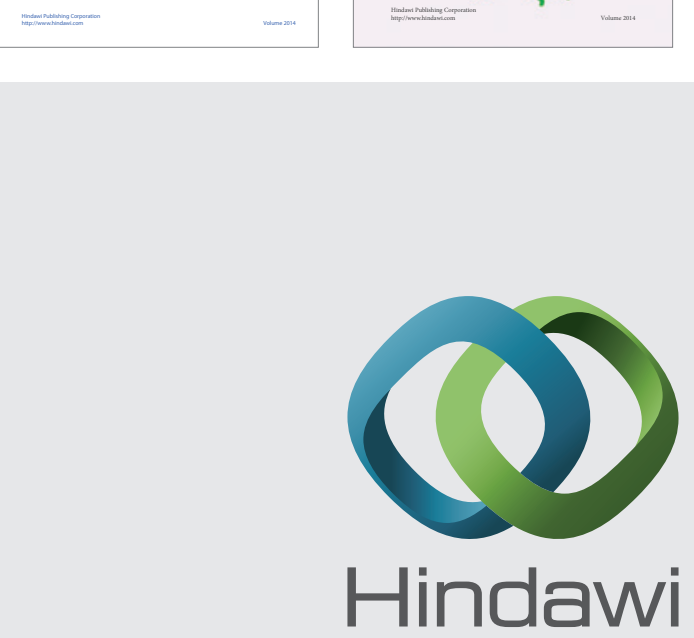

Submit your manuscripts at

http://www.hindawi.com
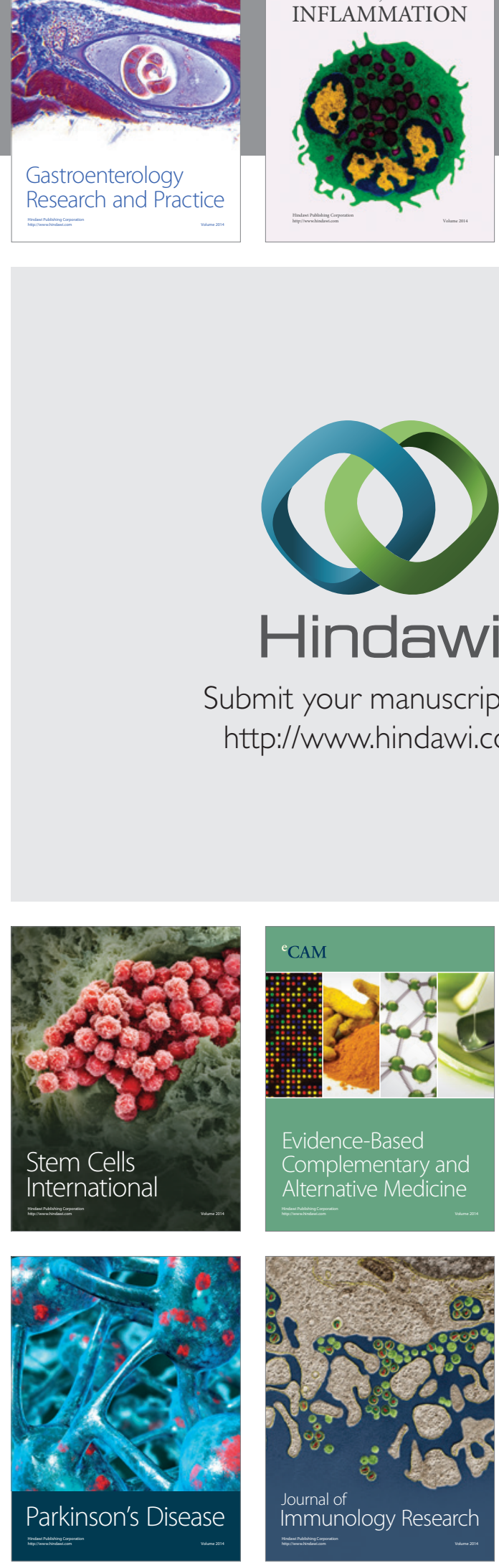

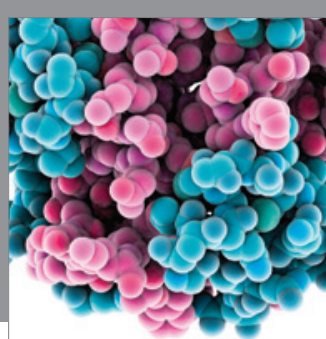

Diabetes Research
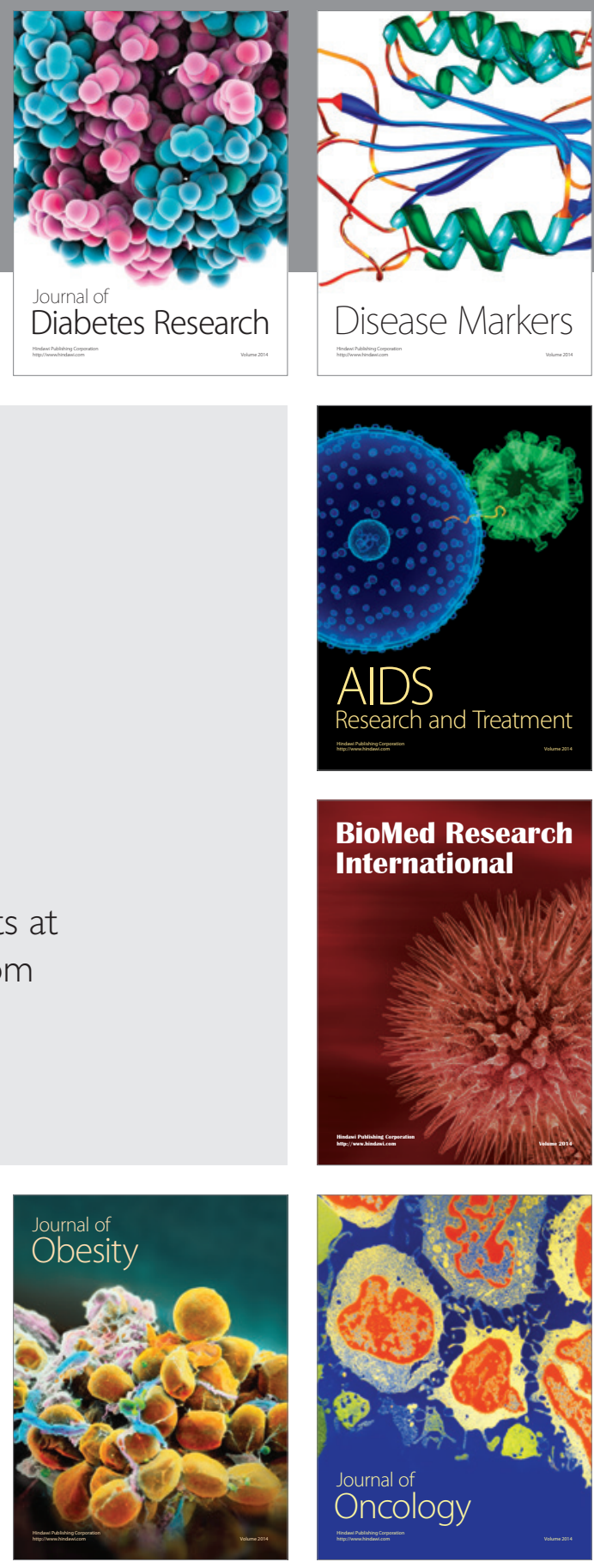

Disease Markers

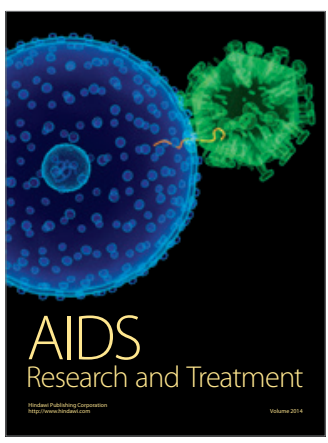

BioMed Research

International
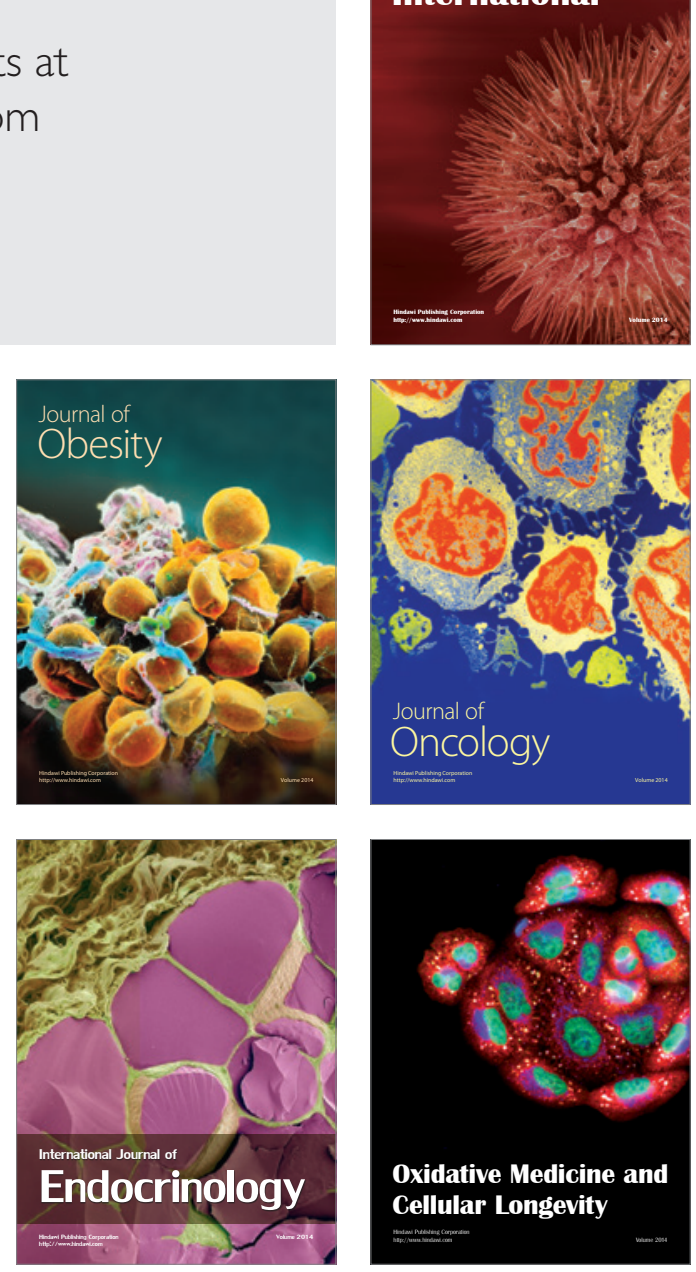